International Journal of Engineering \& Technology, $7(2.33)(2018) 200-205$
International Journal of Engineering \& Technology
SPC
Website: www.sciencepubco.com/index.php/IJET
Research paper

\title{
A survey on internet of things: privacy with security of sensors and wearable network ip/protocols
}

\author{
Subrata Chowdhury ${ }^{1 *}$, Dr. P. Mayilvahanan ${ }^{2}$ \\ ${ }^{1}$ Research Scholar, Department of Computer Application, VISTAS, Pallavaram, Tamil Nadu, India \\ 2 Professor, Dept. of Computer Application, VISTAS, Pallavaram, Tamil Nadu, India \\ *Corresponding author E-mail: mayilkadir@yahoo.com
}

\begin{abstract}
The paper gives the introduction of the IoT, which lay forward the capabilties to detect and link with the world wide web with the connectivity to connect this with the physical objects into a cognate systems. On the other hand some severe concers as the part of the IoT, are been highlighted over the passage gateways of critical personal informations relevants to device and personalized privacy.This survey will summerize and tries to highlight the core issues related to the security threats, and the privacy interest of the IoT.
\end{abstract}

Keywords: Security; Networks Protocols; Sensors; Wearable's, Algorithms.

\section{Introduction}

The IoT in general can be coined as the joining between the virtual and the real world, which means that the machine and the human being direct involvement [1]. Its brings the IoT in the mode continuous growing and evolving, IoT technology is the coming days going to change the way we see the world. Its works with the interconnection of the objects .The connectivity goes at the next level of the machine to machine interactions [2].

IoT plays major roles in the delivering of the different services form the starting of the transportations to banking form the fields of the healthcares to the smart energy productions with the help of the heterogeneous sensors and the networks with the very complex form of the architectures. The efficiency of the data retrievals of the various data objects IoT based services is been developed and deployed in the day to day life [3].

The primary targets of the data processing in been achieved with IoT. Nevertheless most of the medicals aid comes with the cable connections which are very time consuming in terms of the performance and analysis this can cause the misconduct and error in the events. So to overcome this kind of critical issues Remote Health Monitoring Systems has been proposed and here the sensors and networks play the vital roles [4].

\section{IoT perspective and aquirement}

The main key values and the aim of the IoT is to be connected anytime, anyplace with anythings and with anyone,flautlessly using any of the paths/ networks, and any kind of the services [1]. They are the IoM connects with the materials objects of the Internets. for examples its may be the RFID,laser scanners ,globals writings systems ,infrared sensors, and the other Auto ID technology and the informations sensing devices that are been connectify with the various real worls objects for the services of the communations and the exchange of the data. At the last to reach with the smart devices which ca be trackable with the sensors and the loca- tions with GPS and the monitoring faciltyto handle with the networks functions to credible with the infrastructures and the physicals infrastructures cognited with the IoT is been in the needs of the today.

Changes in the Frame of Time.Before [1] we go in the depth of the IoT its will be the advicible to look in to the time frames of the evolutions of the Internets. Its all been started with communications dated back in the 1960, were the network has been set up nad its been possible with the netwroks. In the early 1980 there has been the stacks of the TCP /IP are been introduced. Then there has been the set ups of the commercials networks, there has been the pioneering of the world wide web in short the WWWbecame avaiable in the early 1990, slowly gradually in the 1991, its becomes very popular and generated the rise in the growths of the Internet.The then mobiles devices which been connected with the Internets are been formed with the mobile internet.With the implification of the socializing sites the cocepts of the Iots goes strong.Te next steeps in the IoTs will be the communicatiosn between the machine to machine and which wil be to connects itself to its with the help of the Internet. Its been very impressing and the future of the IotT is very well where the whole world be connected with the Internet and they able to be communicate aslo via internet along the length of the minimal humane interventions. The endlie will be to create a better an safer future for the human beings. and with the simplications of the we like to have a systems which has a Intelligence and the notions of whats we like, what we want and what we needs and which can acts accodinly without any explicitThe main key values and the aim of the IoT is to be connected anytime, anyplace with anythings and with anyone,flautlessly using any of the paths/ networks, and any kind of the services [1]. They are the IoM connects with the materials objects of the Internets. for examples its may be the RFID, laser scanners, globals writings systems, infrared sensors, and the other Auto ID technology and the informations sensing devices that are been connectify with the various real worls objects for the services of the communations and the exchange of the data. At the last to reach with the smart devices which ca be trackable with the sensors and the locations with GPS and the monitoring faciltyto han- 
dle with the networks functions to credible with the infrastructures and the physicals infrastructures cognited with the IoT is been in the needs of the today.

Changes in the Frame of Time.Before [1] we go in the depth of the IoT its will be the advicible to look in to the time frames of the evolutions of the Internets. Its all been started with communications dated back in the 1960, were the network has been set up nad its been possible with the netwroks. In the early 1980 there has been the stacks of the TCP /IP are been introduced. Then there has been the set ups of the commercials networks, there has been the pioneering of the world wide web in short the WWWbecame avaiable in the early 1990 , slowly gradually in the 1991 , its becomes very popular and generated the rise in the growths of the Internet.The then mobiles devices which been connected with the Internets are been formed with the mobile internet.With the implification of the socializing sites the cocepts of the Iots goes strong.Te next steeps in the IoTs will be the communicatiosn between the machine to machine and which wil be to connects itself to its with the help of the Internet. Its been very impressing and the future of the IotT is very well where the whole world be connected with the Internet and they able to be communicate aslo via internet along the length of the minimal humane interventions. The endlie will be to create a better an safer future for the human beings. and with the simplications of the we like to have a systems which has a Intelligence and the notions of whats we like, what we want and what we needs and which can acts accodinly without any explicit instructions.

\section{Achitecture and the basic protocols of IoTs}

The IoT [1] can be classified into three very crucial layers which are the Perceptions, Netwoks and the Applications.he perceptions layers collects the data /informations and establish the physical world. Netwroks layer comes in the middle (Aslo called the wirelss sensors netwroks) which has been liable for the beginning the processings of the infornations, and broadcastings the informations ,sortings and mixing .The topmost applications layers do this services for all the industries.among this layers the middle one plays the most importants role that why its been called the "Central Nervous systems " which is aslo termed as the globals servie of the IoTs, since its been acts as the combing with the upwards aplications layers and makes the link up with the downwards of the perceptual layer.

Numerous baiscs types of the networks includings the mobile/ private networks wired and the wireless networks which are nowadays offering and vouch the necasary connections. IoT protocal stacks "IEEE diagram decription check it later" .Given that the massive amount of the IoT applications howevers will be in the need of the few bits to be send. Its may be sensible to initiate looking into a equable PHY layer ultra low rate transmissions over the very minute midible frequency bands with the accesible advantages of the massive links budgets and thus gradually boost ranges IEEE802.15.4e standards is very suitable for the protocol stacks for IoT as its the advance and latest generations of highly stable and low power MAC protocols.

From the network cicumspect the introductions of the IETF 6Lo WPAN protols systems has been in the effort of the connectings the low power radios to the intranet and then work of IETF ROLL whih in terms allowing for the preferable routings protocols to fulfill the global connectivity. Looking through the perpective of the transport layer the applications, the addition of the IETF CoAP protocol family has been the involved in ensuring that applications within there own do not need to be re-engineered to run on the low levels and power embedded networks.

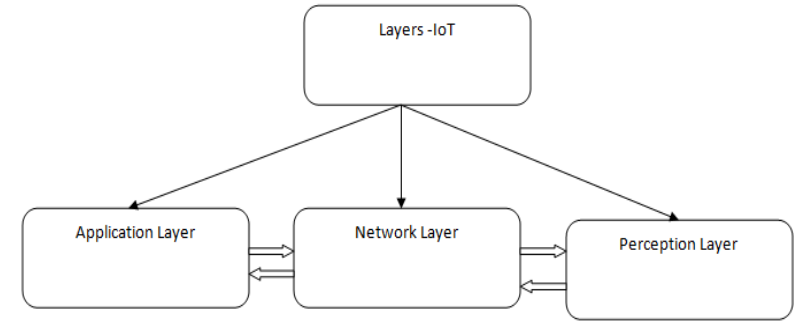

Fig. 1: layers of IoT.

\section{Security and privacy concerns of the data}

With the handful of technology here comes the security concerns, the large amount of data / information which are being generated in the due course of time have a chance to get manipulations or leaked while handling with the hardware devices or software. [2]

\subsection{Confidentiality}

Its acts [2] a firewall which protect from the unauthorized access of the data of the medical field and also protects in keeping encrypted from the being seen.

The IoT status and visions of some well known firms:

Table 1: IoT Status and Vision

\begin{tabular}{ll}
\hline Firms & Status \& Vision \\
\hline \multirow{3}{*}{ Cisco } & $\begin{array}{l}\text { It's been ready to provides combined systems based on the } \\
\text { unrelated networks and its can generate the algorithms for } \\
\text { handlings the massive data traffic originated. }\end{array}$ \\
Google & $\begin{array}{l}\text { Its supports the open source physical web standard for the } \\
\text { IoT } \\
\text { Ticrosoft }\end{array}$ \\
& $\begin{array}{l}\text { The Company has developed a smart business tools which is } \\
\text { capable of the pulling information form the collective data } \\
\text { count. }\end{array}$ \\
\hline
\end{tabular}

\subsection{Integrity}

The data integrity protects the medical data which are not altered and he security brach has not to be there.

\subsection{Authentications}

The introductions of the smart handful of devices has created the heavy potential both for the consumers and the users with this kind of the opportunity the risk is there that the hackers can steal the valuable personal data and the unique information of any product which makes the company vulnerable.

\subsection{Availability}

The availability of the data and the information is very vitals form the consumer point of views, once the device been lost the new device has to streams down the data form online. So once again the medical device which been lost can buy a new device with the same historical data being feed to it. So the patients can use it.

\subsection{Data freshness}

The data freshness include the data freshness and the key freshness because each time the devices will be producing the new data the old data should not repeats as its can damage the credibility of the machine and the patients health jeopardize.

\subsection{Fault tolerance}

The structure or the designs of the devices which are been used in the Medical health has the capability that if there has been any glitch or the fault in the running of the machine it's should immediately generates an alarm of its failure 


\section{IoT healthcares services and applications}

The IoT healthcares services and applications can be classifieds into points the IoT services includes:

Ambient assist livings: The Ambient Assist of living is the mode of giving service to the elderly women and men who are sufferings from the Dementia.

Internets of m- Health: It's an integrated study of the IoT in the field of the medical field and the bio science the checking of the level of the chronic diseases such as the diabetic patients, the invasive of the glucose levels and monitoring of it.

Adverse drug reactions: The injury can caused due to the prolong used of the drugs, the checks of the drugs and strict monitoring of the drugs its adverse effects can be controlled.

Community healthcares: The community healthcare which can be first giving the primary cares and the secondary the hospital care can be given and through the IoT we can monitor it. Centralization of the data the cares extended to the remote villages.

Children health Information: The information of the child can be recorded and the national survey for the populations and the specific information can be gather any disability can be identified the decoding of the information can be investigated.

Wearable Devices: As the world populations is been increasing and parallel with that the aging of the human being there has been the need of the medical aid ,and to overcome that the facility of the wearable technology is evolving itself.

Semantic Medical Access: To develop a semantic healthcare the steps includes to deliver one way window for the medical field which will translate the medical reports and make in a format of oncology and help to solve the queries of the users.

Indirect Emergency Healthcare: The emergency healthcare is been given in the long term responding on the long terms health effects or form prolong suffering of any chemical or pollution of the environments.

Embedded Gateway Configurations: As we know the sensors capabilities of the networks is very limited, they need to connected with the wide area network (WAN), so a gateways has been made to connect themselves with the software .

Embedded context prediction: The ability to give information or services related to the user benefits with the context of the [2].

The services which can be broke into two points that may be the single conditions and the clustered conditions. Further again they can be breakdown into points.

\subsection{Single conditions}

Glucose level sensing: The chorionic diseases patients are regularly checked with the glucose level sensing the CGM (Continuous Glucose Monitoring) is done through real time day and nights, it is inserted in to the skin to measure the levels of the glucose, the electrode is connected with the transmitter which sends the information via wireless radio frequency and monitoring it on the display device.

ECG Monitoring : The ECG sensing network is the central nervous systems of the whole systems, whose main aim is to collection of the physiological data from the body and transmit it to the IoT cloud .The wearable sensors are been used for the data collection so that its do nt crate any huddle in our daily life activities.

Blood Pressure Monitoring: The Intelligent blood pressure monitoring systems is been based on the IoT ,its consists of the intelligent blood pressure monitor, data processing centre, doctor workstations, and the communication facilities. The monitoring device will collect the blood samples and it will send it to the data processing units, in the processing units in the unit it will check the relevance of the data and will format its into graph and sent it to the doctor workstations, the doctor will check the patients report and make it's a summary and sent it to the communication facilities were the patients can check its report.

Body temperature Monitoring: The measuring of the body temperature are been done through the TCP/IP protocols the digital temperature sensors are fitted to the patient's body, the data is been transferred through the dedicated IP on the cloud via the networks wireless modem environments, it will display the data in any form of the graphs as the pie types, bar type, line type its can also be visualize on the screen of an LCD display interfaced to the microcontroller.

Oxygen saturation Monitoring: Oxygen saturations ( $\mathrm{SpO} 2)$ are defined as the ratio of the oxyhemoglobin to the total concentrations of the hemoglobin. Along with this we have to check the heart rate, blood pressure, body temperature and the breathing rate .The SPO2 is a crucial parameter globally used for the detections of the hypoxemia. The SPO2 and the HR are been continuously measure with the help of the wireless devices remotely and monitoring it remotely gives the patients freedom of movements.

\subsection{Clustered conditions}

Rehabilitation System: The growing challenges which are faced by the patients care units and the medical assist teams to serve the number of the people are aging and living with the non communicable diseases and this consequence is very worst.This is the serious call for the policy makers. There has been a rise in the years live in the disability (YLID), the plays the major roles to monitors these patients and the

Medical Managements: The synergic approach that facilitates recommended medical diagnosing. To assure that the benefits are been taken to the lower section of the society , and also the differently able people, sick or emergency patients .Its refers to the preparation of an blueprint and organize the healthcare services and supports this to achieve the goal of medical recovery .

Wheel Chair Managements: The transitions of the transfer from the bed to the wheelchair to shower bench to car seats but when the transfer is difficult or unsafe, which leads to the major problems like risk of the fell down, and skin breakdown, he need for getting down. The IoT sensors attach with this wheelchair can alert the patients and the care unit of the positioning of the patients or elderly. To achieve these goals the Ardino sensors with the thermal imaging concepts are been puts into the patient's body and the GPS are been fitted with the sensors networks on the wheelchairs.

Imminent Healthcare: The first framework that's helps us to characterize digitals IoT systems frameworks which include:

- $\quad$ Edge nodes

- A gateways or a combined cloud

- A back end data analytics which has been operating of the aggregated data for the tend analysis.

The Edge nodes generally collects the raw information with the real time measures such as the various sensors .The wearable fitness devices and the FDA regulated devices such as the glucose level sensors and the ECG monitors are some of the examples of the edge nodes. The valuable information which are been are been collected in raw form are to be processed and the meaningful explanations can be extracted from this.

After thus this the cloud aggregator or the gateways fetches the information through this wearable's and the Wi-Fi, GPS systems positioned the data in the cloud with smart connectivity. On the other hand back-end algorithms run on the embedded chips to give the patients data which are been collected in the short span of time. The frameworks of the data analytics helps us to understands the machine learning algorithms, which leads the artificial intelligence into this entire systems, which helps to transform the data into the actionable insights. The small algorithms are been used for the extractions of the large amounts of the data from the text forms, signals sensors, and the thermal images and the recordings of the patients data to automate and accelerate the diagnostic capabilities Smartphone Healthcare: powerful medical apps which are turning our mobiles into the mobile medical clinics there are so much instructions and guidelines to follows for the betterment of the health. The smart phones connected with the devices which are collecting the medical and the other required medicals data use the smart phones to transfer collect. 


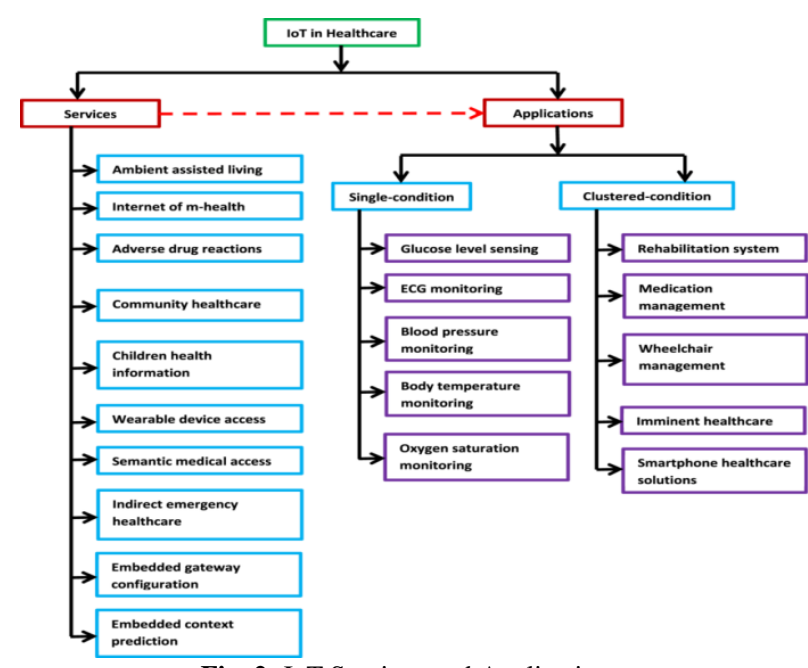

Fig. 2: IoT Services and Applications.

\section{IoT healthcares networks}

The IoT healthcare can be divided into the 3 layers:

Topology Layer: This layer deals with the configurations of the applications and the scenario's and the test cases.

Architecture Layer: Its is the backbone of the proposed systems. The software installations and the connectivity of networks are done here. The Body Sensors Networks (BSN) architectures multiple sensors nodes and the coordinators worn by the patients to checks the electrocardiograms, sensors for the glucose levels the full scheme is designed in the layer.

Platform Layer: This layers deals with the library which stores the valuable information or data which can be used by the medical assistant teams and extract the valuable information. most of the frameworks designed are used for the better understandings of the data and the information comings the protocols and the IP of the gateways .The effectiveness of the module generates a free pool of data which are been used by the data analytic. The environments are bug and error free so that the relevance of the patient's diagnosis and the chart is fault free.

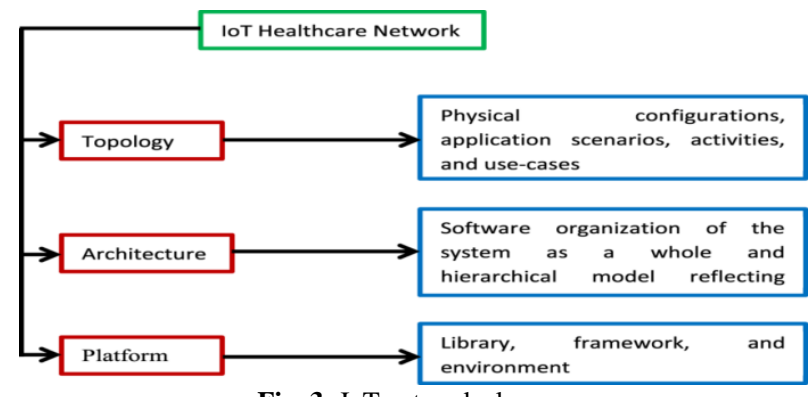

Fig. 3: IoT networks layers.

\section{CoAP protocols}

[8] Constrained Applications Protocols (CoAP) is the special web transfer protocols for the use with the constrained nodes and constrained networks in the Internets of Things. This has been designed specifically for the machine -to-machine (M2M) applications such as the smart energy and the buildings of the automations. The CoAP uses the REST architecture where the stuff are been used by the Universal Resource Identifier (URI) $>$ the main difference between the Http and the CoAP Protocol is that the HTTP protocols is been dependent on the TCP Layer and the CoAP protocols is been dependents on the UDP layer. The format of the messages in the CoAP protocols is that its uses the Request and the Response, using the simple form of the binary, base header format. As we know that CoAP is default to UDP but sometimes it's bound itself with the DTLS which provides the high level of the communications security. In many of the CoAP Ap- plications domain it has the ability to address several of the CoAP cache of the group communications.

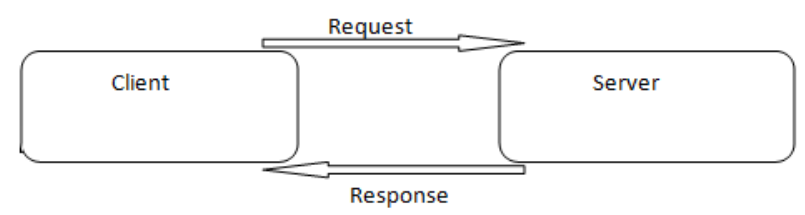

Fig. 4: Client Server Communication in CoAP Protocol.

\section{Electrocardiogram integrated with the IoT}

The elderly people are mainly at the risk of sufferings of the chronic diseases such as the cardiovascular, Alzheimer, Dementia, Parkinson. Most of the medical machines which are been used comes with cable, which are very much time consuming and some time inefficient [6]. Which lead to the misdiagnosing which leads to the death of the patients, so to overcome this problems Remote Health Monitoring Systems is been designed to carry this task at the emergency levels of the patients. The RHMS is based on the IoT, based on the wireless communications and the cloud computing. Based on the category the ECG works on the RHMS and the Wireless Sensors Networks WSN. To procure the limitations of the old systems, so many wirelesses and the portable form of the ECG systems has been developed, getting enkindled by the rapid pace of the advancements in the field of the IoT technology in terms of the Data storage, Wireless communications and the data computations. In fact today's markets, ECG Embedded garments are been launched which send the information remotely and stored into the cloud and the physicians can retrieved that information. In short ECG machine which are coming are easy to handle and fault tolerance and does not have complex design very simple to use by the doctors and the patients. As this devices are mobile.

\section{Advantages of IoT in medical field}

Medical Data Collecting Subsystem: Its main aim is to collect the sample of the data and that is vital for the further diagnosis of the patients which do the analyzing, storing and the formatting of the data [13].

Mechanized Drug Transmission Subsystem: The mechanized drug transmission is based on the patient's symptoms, the subsystems are been collecting the data from the medical data collecting subsystem in need for the search of the medications of the patients, and also for the selection of the medicine. This system creates complete systems for the patients.

Robotics Mechanic Deliver System: This concepts works with the mechanized drug transmissions, its gives the mechanized delivery of the drugs also the payments gateways are been done through the mechanized channels only.

Wireless Sensors Networks Projects: Body sensor which are been used for the monitoring the patients this wireless technology can be used remotely to sensor the patients and also its made possible to monitor the patients with the help of the tiny sensors fitted on the body which will efficient the results also reduce the cost time and the money[11]. In the body sensor network the tiny sensor are been attached to the patients which collects the psychological changes of the body..

BSN Works Done In the Healthcare with IoT Concepts:

CODE BLUE: Its one of the very efficient research based healthcare project which is been developed by the Harvard sensor Network lab in the US this is based on the wireless sensor network. The projects highlights about the sensors which are been fitted to the body of the patients and if any minute changes happed its collects data and its send the data to the computer, PDA notebook, for the further analysis.

ALARM-NET: It's been the brain child of the University of Virginia. Its works on the livings and residential monitoring networks, this network is been used in major robust areas. 
UBIMON: It's been developed by the team of professors in the London Imperial College, the main motto of this project is to calculate the age of the sensors and networks.

MOBICARE: It's used in wider area for the continuous timely monitoring, of the mobiles monitoring systems.

The body sensors network technology is one of the primary technology of which has been develop. The sensor which been fitted to the patient's body are small and low powered and very light weight WSN (Wire Less Network)

\section{Algorithms used}

Followings are the basics main components of the body sensor network:

Body Sensor Network Care Unit (Body Sensor Network Care)

Local Processing Unit (LPU)

Body Sensor Network Server (Body Sensor Network)(11)

Body Sensor Network Care Unit: In the Basic Sensors Networks BSN the sensors which are wearable which is been attached with the patient's body. Each sensor like ECG, Blood pressure are used for monitoring the patients health .The data are been collected in real time.

Local Processing Unit: Local Processing Units means the smart phones, PDA, Laptops, or Embedded chips. It's the Routing between the BSN Server and the BSN link .LPU acts as the routing bridge between the BSN server and the BSN Link. Its uses the Internet connection via the Ethernets or through the GSM kits or the internet's through both the connections.

Body Sensor Network Server: When the BSN Server fetched the information from the LPU then its stores the data into the database and then validate it's with the standard of the physicals data. Its quarterly reviews the data and updates the information into the database. If any difference in the data it's immediately review it with the standard data then check other databases to get the patients family friends and the physician who is monitoring its health and sends the emergency alert messages to their numbers. Algorithms Steps:

Step 1. Patient registration.

Step 2. Login.

Step 3. Insert General information into the database for analyzing.

Step 4: Every $2 \mathrm{sec}$ onwards the patients reading are been collected and stored.

Step 5: Collected data is reviewed with the previous collected data. Step 6. If any abnormality got during the review of the data collected from the patient bodies immediately find the numbers of the patients' family, relative, nearest ones and the consultant physician under whom he is being monitored.

Step 7: Automatically an alert message should be send to the members of the family the nearest ones, friends and the Physician.

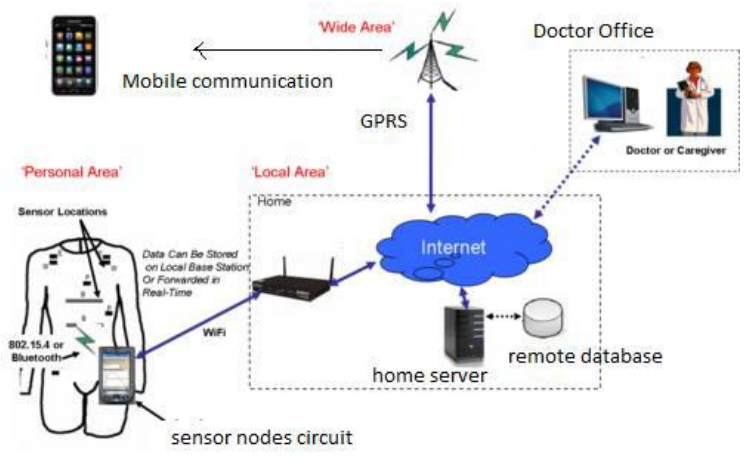

Fig. 5: The System Architecture.

\section{Conclusion}

Providing security is a challenging task and maintaining a secure and error free network for the sensor and wearable is very problematic. Everyday new errors are generated and troubleshooting those errors are quite challenging. IoT healthcare benefits reached to everyone which is affordable, low cost and user-friendly, and less complex is the goal of this survey. The networks family which has its nodes spread over the thousands of the connections are to fault and bug free for the smooth running information is to achieve this the protocols and the routing has to bind up in such a way that the data or the information passes through its is safe and secure, the set up models are well advanced and cheap costs to be make sure, that the Health care's reaches to them who are in urgent need like the elderly, disabled and the remote areas were the IoT can play the vital role.

\section{References}

[1] J.Sathish Kumar and Dhiren R Patel "Survey on Internets of Things: Security and Privacy Issues" International Journal of Computer Applications (0975 - 8887) Volume 90 - No 11, March 2014

[2] S. M. RIAZUL ISLAM1, (Member, IEEE), DAEHAN KWAK2, MD. HUMAUN KABIR1, MAHMUD HOSSAIN3, AND KYUNG-SUP KWAK1, (Member, IEEE) "The Internets of Things for HealthCare: Comprehensive Study" IEEE Access Date of publication June 1, 2015, date of current version June 4, 2015.

[3] Shu-yuan Ge, Seung-Man Chun, Hyun-Su Kim and Jong-Tae Park "Design and Implementation of Interoperable IoT Healthcare System Based on International Standards" 13th IEEE Annual Consumer Communications \&Networking Conference (CCNC) ,2016.

[4] Arijit Ukil and Soma Bandyoapdhyayand Chetanya Puri and Arpan Pal "IoT Healthcare Analytics: The Importance of Anomaly Detection" 30th International Conference on Advanced Information Networking and Applications IEEE 2016.

[5] KUO-HUI YEH, (Senior Member, IEEE) “A Secure IoT-Based Healthcare System With BodySensor Networks" IEEE Access, Date of publication December 9, 2016, date of current version January $27,2017$.

[6] Wan Aida Nadia binti Wan Abdullah, N. Yaakob, R Badlishah, A Amir, Siti Asilah binti Yah,ENAC," On the effectiveness of Congestion ControlMechanisms for Remote Healthcare Monitoring System in IoT Environment - A Review" 3rd International Conference on Electronic Design (ICED), August 11-12, 2016, Phuket, Thailand 2016

[7] Ons Bibani, Carla Mouradian, Sami Yangui†, Roch H. Glitho, Walid Gaaloul, Nejib Ben Hadj-Alouane, Monique J. Morrow Paul A. Polakos "A Demo of IoT Healthcare Application Provisioning in Hybrid Cloud/Fog Environment" IEEE 8th International Conference on Cloud Computing Technology and Science 2016.

[8] Shu-yuan Ge, Seung-Man Chun, Hyun-Su Kim and Jong-Tae Park' Design and Implementation of Interoperable IoT Healthcare System Based on International Standards "13th IEEE Annual Consumer Communications \& Networking Conference (CCNC) 2016.

[9] Dejana Ugrenovic ,Gordana Gardasevic “ CoAP Protocols for Web -based monitoring In IoT healthacare applications" 23 rd Telecommunications forum TELFOR 2015

[10] "https://en.wikipedia.org/wiki/Constrained_Application_Protocol]."

[11] Uttam U. Deshpande, Milan A. Kulkarni" IoT based Real Time ECG Monitoring System using Cypress WICED” (An ISO 3297: 2007 Certified Organization) Website: www.ijareeie.com Vol. 6 , Issue 2, February 2017.

[12] Deshpande Niranjan R 1, Vadane Pandurang M 2 , Sangle Sagar D 3 , Prof. Dighe M. S.4', A IOT-based Modern Healthcare System Using Body Sensor Network (BSN) " International Journal of Innovative Research in Computer and Communication Engineering (An ISO 3297: 2007 Certified Organization) Vol. 4, Issue 11, November 2016.

[13] Ons Bibanit\&, Carla Mouradian†, Sami Yangui†, Roch H. Glitho†, Walid Gaaloul\$, Nejib Ben Hadj-Alouane§, Monique J. Morrow , Paul A. Polakos", A Demo of IoT Healthcare ApplicationProvisioning in Hybrid Cloud/Fog Environment " IEEE 8th International Conference on Cloud Computing Technology and Science 2016

[14] M. Hadjem, O. Salem, F. Nait-Abdesselam, "An ECG monitoringsystem for prediction of cardiac anomalies using WBAN," IEEE 16thInternational Conference on e-Health Networking, Applications and Services (Healthcom), pp. 441-446, 2014.

[15] T. Musha, H. Matsuzaki, Y. Kobayashi, Y. Okamoto, M. Tanaka, T.Asada, T., "EEG Markers for Characterizing Anomalous Activities ofCerebral Neurons in NAT (Neuronal Activity Topography) Method,"IEEE Transactions on Biomedical Engineering, vol.60, no.8, pp. 2332-2338, Aug. 2013.

[16] C. C. Aggarwal, "Outlier Analysis," Springer Publication, 2013. 
[17] P. Horn, L. Feng, Y. Li, and A. J. Pesce, "Effect of Outliers andNonhealthy Individuals on Reference Interval Estimation," ClinicalChemistry, pp. 2137-2145, 2001.

[18] S. Roberts, "Extreme Value Statistics for Novelty Detection inBiomedical Signal Processing," International Conference on Advancesin Medical Signal and Information Processing. pp. 166-172, 2002.

[19] M. Prastawa, E. Bullitt, S. Ho, and G. Gerig, "A Brain TumorSegmentation Framework based on Outlier Detection, Medical ImageAnalysis," pp. 275-283, 2004.

[20] https://www.physionet.org/physiobank/database/mitdb/

[21] J. Chen, et al., "Blue scale: Early detection of impending congestiveheart failure events via wireless daily self-monitoring," IEEE HealthcareInnovation Conference (HIC), pp. 63-66, 8-10 Oct. 2014.

[22] A. Ukil, S. Bandyopadhyay, C. Puri, and A. Pal, "Heart-trend: anaffordable heart condition monitoring system exploiting morphologicalpattern," IEEE International Conference on Acoustics, Speech andSignal Processing (ICASSP), 2016 (accepted).

[23] A. Ukil, S. Bandyopadhyay, J. Joseph, V. Banahatti, and S. Lodha,Negotiation-based privacy preservation scheme in internet of thingsplatform," IEEE International Conference on Security of Internet of Things, pp. 75- 84, 2012.

[24] L. Clifton, D.A. Clifton, M.A.F. Pimentel, P.J. Watkinson, and L.Tarassenko, "Predictive monitoring of mobile patients by combiningclinical observations with data from wearable sensors," IEEE Journal ofBiomedical and Health Informatics, vol.18, no.3, pp.722730, May2014.

[25] A. Ukil, S. Bandyopadhyay, A. Bhattacharyya, A. Pal, T. Bose., "Auth-Lite: Lightweight M2M authentication reinforcing DTLS for CoAP," inPervasive Computing and Communications Workshops (PERCOMWorkshops), vol., no., pp.215-219, 24-28 March 2014.

[26] K. Raghuraman, M. Senthurpandian, M. Shanmugasundaram, M.Bhargavi, V. Vaidehi, "Online incremental learning algorithm foranomaly detection and prediction in health care," International Conference on Recent Trends in Information Technology (ICRTIT), 2014.

[27] S. Bandyopadhyay, A. Ukil, C. Puri, A. Pal, R.Singh, T. Bose "Demo:IAS: Information analytics for sensors," 13th ACM Conference onEmbedded Networked Sensor Systems, Sensys 2015.

[28] A. Ukil, S. Bandyopadhyay, A. Bhattacharyya, A. Pal., "Lightweight security scheme for vehicle tracking system using CoAP," Ubicomp-ASPI Workshop. 